\title{
Neonatal Maternal Deprivation Followed by Adult Stress Enhances Adrenergic Signaling to Advance Visceral Hypersensitivity
}

\author{
Wan-Jie Du ${ }^{1} \cdot$ Shufen $\mathrm{Hu}^{1} \cdot \mathrm{Xin} \mathrm{Li}^{1} \cdot$ Ping-An Zhang ${ }^{1,2} \cdot$ Xinghong Jiang $^{1} \cdot$ \\ Shan-Ping $\mathrm{Yu}^{3} \cdot$ Guang-Yin $\mathrm{Xu}^{1,2}$
}

Received: 27 July 2018/Accepted: 26 September 2018/Published online: 17 December 2018

(C) The Author(s) 2018

\begin{abstract}
The pathophysiology of visceral pain in patients with irritable bowel syndrome remains largely unknown. Our previous study showed that neonatal maternal deprivation (NMD) does not induce visceral hypersensitivity at the age of 6 weeks in rats. The aim of this study was to determine whether NMD followed by adult stress at the age of 6 weeks induces visceral pain in rats and to investigate the roles of adrenergic signaling in visceral pain. Here we showed that NMD rats exhibited visceral hypersensitivity 6 $\mathrm{h}$ and $24 \mathrm{~h}$ after the termination of adult multiple stressors (AMSs). The plasma level of norepinephrine was significantly increased in NMD rats after AMSs. Whole-cell patch-clamp recording showed that the excitability of dorsal root ganglion (DRG) neurons from NMD rats with AMSs was remarkably increased. The expression of $\beta_{2}$ adrenergic receptors at the protein and mRNA levels was markedly higher in NMD rats with AMSs than in rats with NMD alone. Inhibition of $\beta_{2}$ adrenergic receptors with propranolol or butoxamine enhanced the colorectal distention threshold and application of butoxamine also reversed the enhanced hypersensitivity of DRG neurons. Overall, our data demonstrate that AMS induces visceral
\end{abstract}

Wan-Jie Du and Shufen Hu have contributed equally to this work.

Guang-Yin $\mathrm{Xu}$

guangyinxu@suda.edu.cn

1 Laboratory for Translational Pain Medicine, Institute of Neuroscience, Soochow University, Suzhou 215123, China

2 Center for Translational Medicine, The Zhangjiagang Affiliated Hospital of Soochow University, Zhangjiagang 215600, China

3 Department of Anesthesiology, Emory University School of Medicine, Atlanta, GA 30307, USA hypersensitivity in NMD rats, in part due to enhanced $\mathrm{NE}-\beta_{2}$ adrenergic signaling in DRGs.

Keywords Irritable bowel syndrome $\cdot$ Dorsal root ganglion $\cdot$ Norepinephrine $\cdot$ Visceral pain $\cdot$ Stress

\section{Introduction}

Irritable bowel syndrome (IBS) is a common gastrointestinal disease characterized by disorders of intestinal motility and accompanied by chronic abdominal pain [1-3]. The treatment of chronic abdominal pain is difficult [4]. Research on this disease is progressing slowly due to the lack of suitable animal models. Although the currently available research provides insights into the processing and regulation of chronic pain [5, 6], the precise pathophysiology of IBS has not been fully elucidated and effective strategies for treating the primary symptoms are not available $[1,7,8]$. Our previous studies have shown that neonatal maternal deprivation (NMD) can induce chronic visceral pain in adult rats at the age of 7 weeks but not at 6 weeks $[9,10]$. It seems that the age of 7 weeks is an important time point for the development of visceral hypersensitivity in rats with NMD. However, it is unknown whether 6-week-old NMD rats are more sensitive to environmental stimuli than age-matched controls.

A growing body of evidence has demonstrated that severe adverse environmental factors such as stress might be a stimulus to generate visceral hypersensitivity at the adult age in humans [11, 12] and animals [13-15]. For example, repetitive water-avoidance stressors for 10 days induce visceral hypersensitivity in rats and mice [16]. Heterotypic intermittent stress for 9 consecutive days induces visceral hypersensitivity immediately after 
termination of the last stressors [14, 17]. However, single or mild stressors do not induce visceral hypersensitivity in healthy adult animals. In the present study, we designed a stress protocol to determine whether one stressor or mild stressors could induce visceral pain in NMD rats at the age of 6 weeks.

Adrenergic signaling plays many important roles in the nervous system to regulate stress responses [18-20]. Adrenergic receptors (ARs) are classically divided into two main groups: $\alpha$ - and $\beta$-adrenoceptors. The $\alpha$-adrenoceptors include $\alpha_{1 \mathrm{~A}}, \alpha_{1 \mathrm{~B}}, \alpha_{1 \mathrm{D}}, \alpha_{2 \mathrm{~A}}, \alpha_{2 \mathrm{~B}}$, and $\alpha_{2 \mathrm{C}}$ subtypes, and $\beta$-adrenoceptors into $\beta_{1}, \beta_{2}$, and $\beta_{3}$ subtypes [21]. They have been reported to be expressed in primary sensory neurons with their cell bodies located in the dorsal root ganglia (DRGs) [22, 23]. Previous studies have suggested that the $\beta_{1}$ and $\beta_{2}$ subtypes are involved in the adrenergic activation [24] that may play a role in colonic transit. The $\beta_{2}$ ARs are reported to produce a hyperalgesic state in rats $[22,25,26]$. The $\beta_{3}$ ARs, mainly expressed in brown and white adipose tissue, regulate energy metabolism and thermogenesis [27]. Besides, other studies have shown that the adrenergic system plays a role in the visceral pain caused by chronic stress [13]. Recently, we have reported that adrenergic $\beta_{2}$ receptors mediate visceral pain evoked by heterotypic intermittent stress in rats [14]. However, whether adrenergic signaling in primary sensory neurons participates in visceral pain of NMD rats at the age of 6 weeks after additional adult stress is unknown.

Thus, we designed this study to test the hypothesis that adrenergic activation plays a crucial role in the switch of NMD rats at the age of 6 weeks from no pain status to pain hypersensitivity induced by stress in adulthood.

\section{Materials and Methods}

\section{Animals}

We used male Sprague-Dawley rats in the present experiments. The experimental protocol was approved by the Institutional Animal Care and Use Committee of Soochow University. Animal care and handling were strictly in accordance with the regulations and guidelines of the International Association for the Study of Pain.

\section{Adult Stress Protocol to Induce Visceral Pain}

The stress protocol to induce chronic visceral hyperalgesia is shown in Fig. 1. This protocol had two parts, NMD and stress in adulthood. The adult stress was divided into an adult single stressor (ASS), cold-restraint stress (CRS), for $45 \mathrm{~min}$, and adult multiple stressors (AMSs) consisting of CRS for $45 \mathrm{~min}$, forced-swimming stress (FSS) for $20 \mathrm{~min}$, and water-avoidance stressor (WAS) for $60 \mathrm{~min}$. The interval between each stressor was $60 \mathrm{~min}$. NMD was imposed as described previously [9, 10, 28]. In brief, pups were separated from the maternity cages and placed in different cages with an electric blanket to maintain body temperature at $\sim 32{ }^{\circ} \mathrm{C}$ for $3 \mathrm{~h}$ every day from postnatal days 2 to 15 . After the $3 \mathrm{~h}$ of separation, pups were returned to the dam cages. Littermates in the control group were not handled and were kept in the maternity cages with their dam. Both groups were exposed to an ASS or AMSs on postnatal day 42 (Fig. 1). All experiments were performed $6 \mathrm{~h}$ or $24 \mathrm{~h}$ after termination of last stressor unless indicated otherwise. Multiple batches of rats at the age of 6 weeks were used in the present study. The numbers of animals in each group were as follows: control group, 15; NMD group, 17; CON + ASS group, 13; NMD + ASS group, 16; CON + AMS group, 38; and NMD + AMS group, 68 .

\section{Measurement of Behavioral Responses to Graded Colorectal Distention (CRD)}

Chronic visceral hyperalgesia was assessed by grading the behavioral response of rats to CRD at the age of 6 weeks based on previous publications $[9,10,20]$. All behavioral tests were performed in a blinded manner.

\section{Drug Administration}

In the behavioral experiments, $5 \mathrm{mg} / \mathrm{kg}$ butoxamine (BUTO, a $\beta_{2}$ antagonist; Sigma, St. Louis, MO), $3 \mathrm{mg} / \mathrm{kg}$ propranolol (PROP, a norepinephrine $\beta$ receptor antagonist; Sigma) or $3 \mathrm{mg} / \mathrm{kg}$ phentolamine (PHEN, an $\alpha$ receptor antagonist; Sigma) dissolved in $0.9 \%$ normal saline (NS) was intraperitoneally injected into AMS rats once for behavioral experiments and once daily for 7 consecutive days for patch-clamp recordings and western blotting. The drug concentrations used were based on our previous study and reports from other groups [14, 20].

\section{Measurement of Norepinephrine (NE) in Blood Plasma}

Blood samples were collected from the trunk into centrifuge tubes containing $0.45 \%$ citric acid and $2.5 \%$ sodium citrate at euthanasia by decapitation. After refrigerated centrifugation, the supernatant was quickly aliquoted and stored at $-80{ }^{\circ} \mathrm{C}$ for experiments. NE levels in the plasma were determined using an enzyme immunoassay kit from Abnova (Norepinephrine ELISA Kit), as previously described [20]. 


\section{Experimental Protocol}

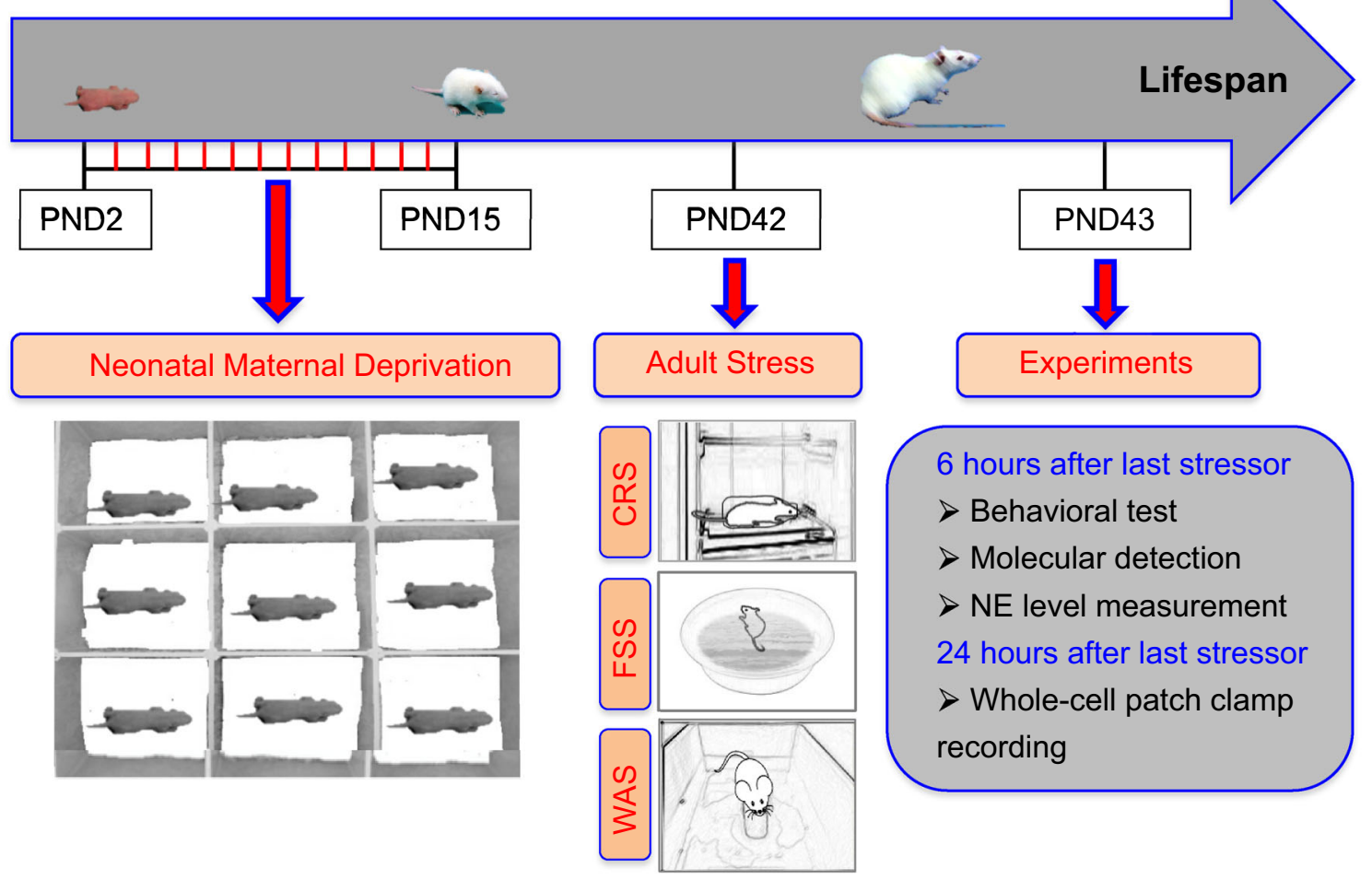

Fig. 1 Cartoon showing the experimental protocol for inducing visceral hypersensitivity. This protocol had two parts: neonatal maternal deprivation (NMD) and adult stress. NMD was imposed from postnatal day (PND) 2 to 15. The adult stress was of two kinds: adult single stressor (ASS): cold-restraint stress (CRS) for $45 \mathrm{~min}$, and adult multiple stressors (AMSs): CRS for $45 \mathrm{~min}$, forced-

\section{Western Blotting}

DRGs at $\mathrm{T}_{13}-\mathrm{L}_{2}$ from AMS-treated control or NMD rats were collected to measure the protein levels of $\beta_{1}, \beta_{2}$, and $\beta_{3}$ receptors. DRGs at $T_{13}-L_{2}$ from control, NMD, and ASS-treated control or NMD rats were collected to measure the protein levels of $\beta_{2}$ receptors. The antibodies used were anti- $\beta_{1}$ (1:500, Santa Cruz Biotechnology, Inc.), anti- $\beta_{2}$ (1:500, Santa Cruz Biotechnology, Inc.), anti- $\beta_{3}$ (1:500, Santa Cruz Biotechnology, Inc.), and anti-GAPDH (1:2000, Hangzhou Goodhere Biotechnology). Band density was measured using ImageJ software. The $\beta_{1}, \beta_{2}$, and $\beta_{3}$ expression were normalized to GAPDH as described previously $[17,20,29,30]$.

\section{Real-Time qPCR}

Total RNAs were extracted from DRGs $\left(\mathrm{T}_{13}-\mathrm{L}_{2}\right)$ from control, AMS, and NMD rats with TRIzol (Ambion, Shanghai, China). cDNA was synthesized from total RNA using a reverse transcription kit (Transgen Biotech, Beijing, China) according to the supplier's instructions. The swimming stress (FSS) for $20 \mathrm{~min}$, and water-avoidance stress (WAS) for $60 \mathrm{~min}$. Behavioral testing was performed $6 \mathrm{~h}$ and $24 \mathrm{~h}$ after the termination of the last stressor. Molecular detection and norepinephrine (NE) measurements were performed $6 \mathrm{~h}$ after the termination of the last stressor. Whole-cell patch-clamp recordings were made $24 \mathrm{~h}$ after termination of the last stressor.

primers are as follows: $\beta_{2}$ forward $5^{\prime}$ GGTTGGGCTATGTCAACTCTG- $3^{\prime}$, reverse $5^{\prime}$ GTCTGTCCTACCGTTGCTGTT-3'; gapdh (internal control) forward 5'-TGGAGTCTACTGGCGTCTT-3', reverse 5'-TGTCATATTTCTCGTGGTTCA-3'. Control reactions were carried out without cDNA templates.

\section{Dissociation of DRG Neurons and Whole-Cell Patch-Clamp Recordings}

Rats from AMS-treated NMD or control ( $\sim 6$ weeks) were sacrificed by decapitation. The detailed procedures for the acute isolation of DRG neurons and patch clamp recordings were as previously reported $[14,20,31]$. The dissecting solution contained (in mmol/L): $130 \mathrm{NaCl}, 5$ $\mathrm{KCl}, 2 \mathrm{KH}_{2} \mathrm{PO}_{4}, 1.5 \mathrm{CaCl}_{2}, 6 \mathrm{MgSO}_{4}, 10$ glucose, and 10 HEPES, pH 7.2 with osmolarity 305 mOsm. For the patchclamp recordings, normal external solution contained (in $\mathrm{mmol} / \mathrm{L}$ ): $130 \mathrm{NaCl}, 5 \mathrm{KCl}, 2 \mathrm{KH}_{2} \mathrm{PO}_{4}, 2.5 \mathrm{CaCl}_{2}, 1$ $\mathrm{MgCl}_{2}, 10$ HEPES, 10 glucose, $\mathrm{pH}$ adjusted to 7.2 with $\mathrm{NaOH}$, osmolarity 295-300 mOsm. The pipette solution contained (in mmol/L): $140 \mathrm{~K}$-gluconate, $10 \mathrm{NaCl}, 10$ 
HEPES, 10 glucose, 5 EGTA, $1 \mathrm{CaCl}_{2}$, pH 7.25 adjusted with $\mathrm{KOH}$; osmolarity $292 \mathrm{mOsm}$.

\section{Data Analysis}

All data are presented as mean \pm SEM. Statistical testing was performed using OriginPro 8 (OriginLab, Northampton, MA). Normality was first checked for all data before analysis. Significance was determined using the twosample $t$ test, Mann-Whitney test, Mann-Whitney test following Friedman ANOVA, or Tukey's post-hoc test following two-way repeated measures ANOVA. $P<0.05$ was considered to be statistically significant.

\section{Results}

\section{AMS Induces Visceral Hypersensitivity in NMD Rats}

Chronic visceral hyperalgesia was assessed as the abdominal withdrawal reflex (AWR) score and distension threshold (DT) in response to CRD at the age of 6 weeks. The NMD rats did not exhibit chronic visceral hyperalgesia at 6 weeks (Fig. 2A, B), consistent with previous studies $[8,29,30]$. Besides, ASS also did not induce chronic visceral hyperalgesia $6 \mathrm{~h}$ after CRS exposure in NMD rats at 6 weeks (Fig. 2C, D). However, the AWR scores increased significantly and DT decreased markedly after AMS exposure (Fig. 2E-H). The AWR scores were $1.50 \pm$ $0.16,2.60 \pm 0.19,3.40 \pm 0.19$, and $3.90 \pm 0.10$ in the NMD + AMS rats $(n=5$ rats $)$ and $0.25 \pm 0.14,1.88 \pm$ $0.13,2.75 \pm 0.25$, and $3.00 \pm 0.20$ in the CON + AMS rats $(n=5$ rats $)$ at $20 \mathrm{mmHg}, 40 \mathrm{mmHg}, 60 \mathrm{mmHg}$, and 80 $\mathrm{mmHg}$ distention pressure $6 \mathrm{~h}$ after AMS exposure, respectively. The AWR scores were $1.25 \pm 0.43,2.88 \pm$ $0.13,3.13 \pm 0.13,3.50 \pm 0.20$ in the NMD + AMS rats ( $n=4$ rats) and $0.00 \pm 0.00,1.50 \pm 0.20,2.13 \pm 0.13,3.00$ \pm 0.00 in the $\mathrm{CON}+\mathrm{AMS}$ rats $(n=4$ rats $)$ at $20 \mathrm{mmHg}$, $40 \mathrm{mmHg}, 60 \mathrm{mmHg}$ and $80 \mathrm{mmHg}$ distention pressure 24 $\mathrm{h}$ after AMS exposure, respectively. In addition, the DT was $38.33 \pm 1.55 \mathrm{mmHg}(n=5$ rats $)$ and $23.33 \pm 1.41$ mmHg ( $n=5$ rats) $6 \mathrm{~h}$ after AMS exposure in the $\mathrm{CON}+$ AMS rats and NMD + AMS rats (Fig. 2F); and was $43.17 \pm 3.02 \mathrm{mmHg}(n=4$ rats $)$ and $21.17 \pm 0.57$ mmHg $(n=4$ rats) $24 \mathrm{~h}$ after AMS exposure in the $\mathrm{CON}+$ AMS rats and NMD + AMS rats (Fig. 2H). After statistical analysis, there was a remarkable difference in AWR scores and DT between CON + AMS and NMD + AMS rats (AWR scores, $P<0.05$, Mann-Whitney test following Friedman ANOVA; DT, $P<0.001$, twosample $t$ test). These data showed that the AWR scores were markedly greater in the NMD + AMS groups at 20
$\mathrm{mmHg}, 40 \mathrm{mmHg}, 60 \mathrm{mmHg}$, and $80 \mathrm{mmHg}$ distention pressures than those of age-matched CON + AMS rats $6 \mathrm{~h}$ and $24 \mathrm{~h}$ after AMS exposure. Meanwhile, the DT was greatly decreased in NMD + AMS rats compared with agematched CON + AMS rats $6 \mathrm{~h}$ and $24 \mathrm{~h}$ after AMS exposure, indicating that AMS induced visceral pain in NMD rats at the age of 6 weeks.

\section{AMS Increases NE Concentration in Blood Plasma of NMD Rats}

$\mathrm{NE}$ is one of the important molecules involved in the regulation of stress responses $[18,20,32]$. In the present experiments, we demonstrated that there was no significant difference in NE concentrations in the plasma between control and NMD groups $(P>0.05$, two-sample $t$ test, Fig. 3A). There was also no significant difference in NE concentration in the plasma between the CON + ASS and NMD + ASS groups $(P>0.05$, two-sample $t$ test, Fig. 3B). However, there was a significant difference in $\mathrm{NE}$ concentrations in the plasma between CON + AMS and NMD + AMS rats, indicating that AMS remarkably increased the NE levels $(P<0.01$, two-sample $t$ test, Fig. 3C).

\section{AMS Enhances Excitability of Colon DRG Neurons in NMD Rats}

To determine the effects of NMD + AMS on the excitability of colon DRG neurons, whole-cell patch clamp recordings were carried out. The resting membrane potentials of $\mathrm{T}_{13}-\mathrm{L}_{2}$ DRG neurons were $-49.25 \pm 1.92 \mathrm{mV}(n=$ 8 cells $)$ and $-46.3 \pm 1.59 \mathrm{mV}(n=10$ cells $)$ for $\mathrm{CON}+\mathrm{AMS}$ and $\mathrm{NMD}+\mathrm{AMS}$ rats, respectively (Fig. 4A). After statistical analysis, there was no significant difference in resting membrane potentials between $\mathrm{CON}+$ AMS and NMD + AMS rats $(P>0.05$, two-sample $t$ test). Rheobase and firing patterns in response to current stimulation were also recorded. The average rheobases of colon DRG neurons were $56 \pm 9.6 \mathrm{pA}(n=8$ cells) from $\mathrm{CON}+\mathrm{AMS}$ rats and $13 \pm 2.1 \mathrm{pA}(n=10$ cells $)$ from the corresponding NMD + AMS rats (Fig. 4B, $P<0.01$, Mann-Whitney test). The number of action potentials (APs) in response to $2 \times$ rheobase current stimulation was significantly increased in DRG neurons from the NMD + AMS group (Fig. $4 \mathrm{C}$ and D, $P<0.05$, twosample $t$ test). However there was no significant difference in the number of APs evoked by $3 \times$ rheobase current stimulation (Fig. 4C and D, $P>0.05$, two-sample $t$ test). In addition, we counted the numbers of APs induced by 100 pA, 300 pA and 500 pA ramp current stimulation (Fig. 4E and $\mathrm{F}$ ). The numbers of APs in response to $100 \mathrm{pA}, 300$ $\mathrm{pA}$, and $500 \mathrm{pA}$ ramp current stimulation differed 


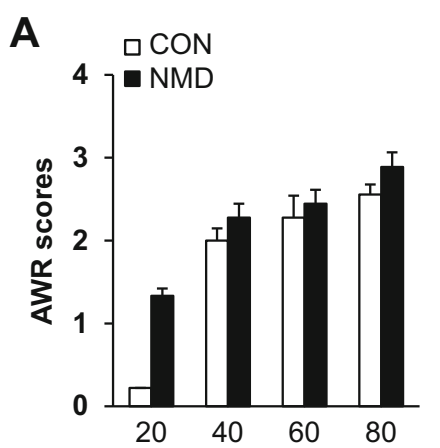

Distension pressure $(\mathrm{mmHg})$
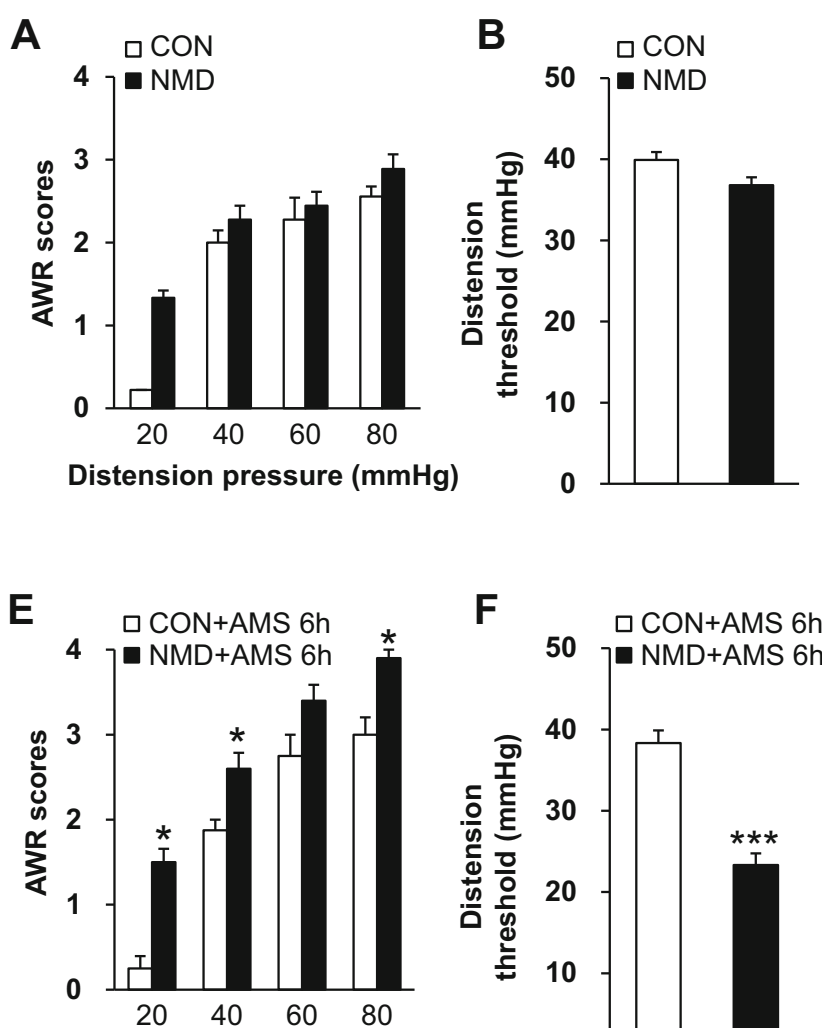

Distension pressure $(\mathrm{mmHg})$
F $\quad \square$ CON+AMS $6 \mathrm{~h}$

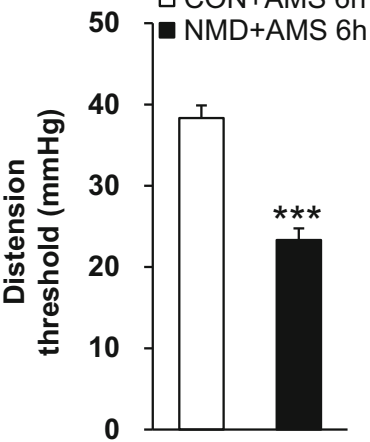

Fig. 2 Multiple stressors induced chronic visceral hyperalgesia in NMD rats at 6 weeks. A, B NMD did not induce changes of distention threshold and AWR scores in response to colorectal distention (CRD) in 6 weeks, compared with controls. C, D NMD + ASS did not induce changes of distention threshold and AWR scores in response to CRD 6 h after ASS exposure, compared with CON + ASS rats. E, F NMD + AMS significantly reduced the distention threshold and
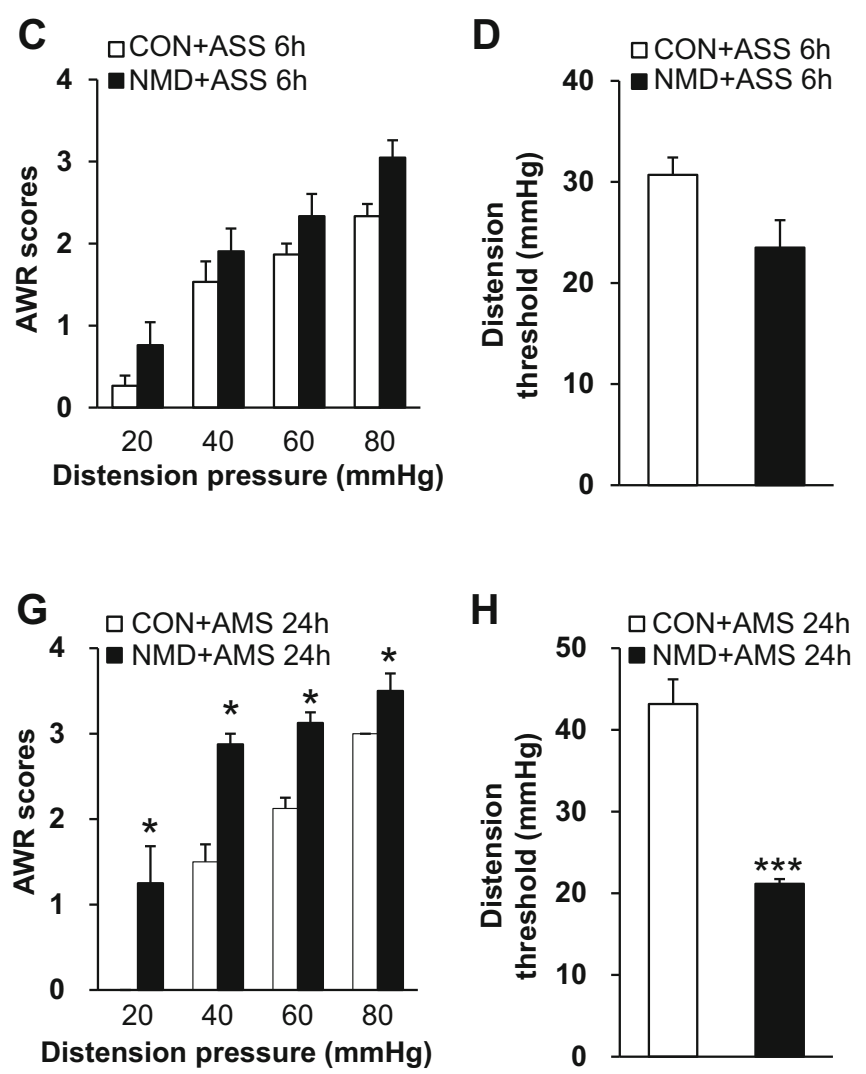

increased AWR scores $6 \mathrm{~h}$ after AMS exposure when compared with CON + AMS. G, H NMD + AMS significantly reduced the distention threshold and increased AWR scores $24 \mathrm{~h}$ after AMS exposure compared with CON + AMS. AWR scores, $* P<0.05$, MannWhitney test following Friedman ANOVA; DT, $* * * P<0.001$, twosample $t$ test.
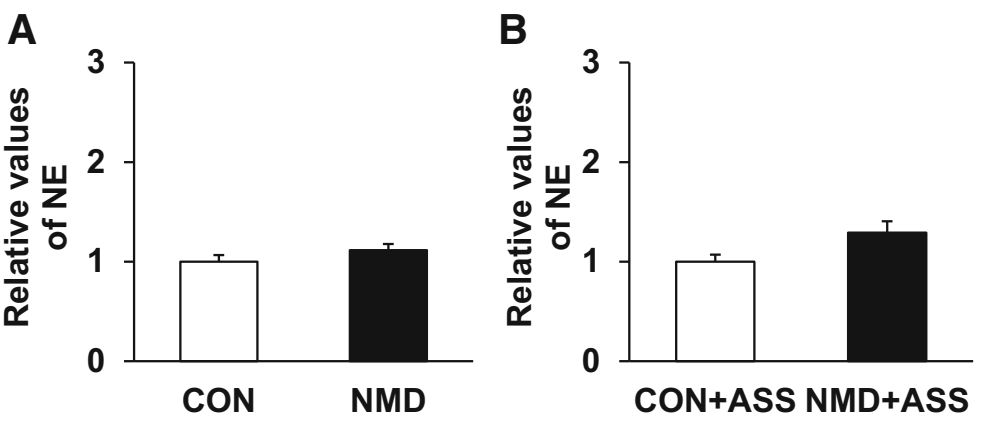

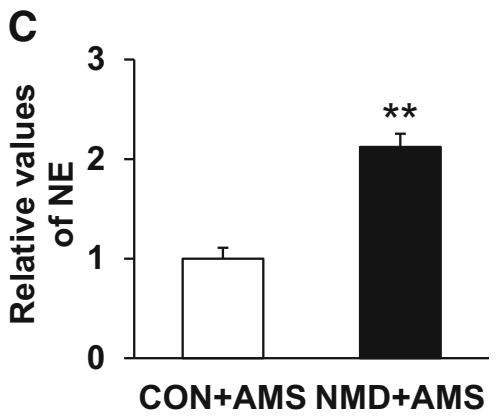

Fig. 3 Multiple stressors increased norepinephrine concentration in blood plasma of NMD rats at 6 weeks. A NMD did not significantly alter the NE concentration compared with controls. B NMD + ASS

significantly between CON + AMS and NMD + AMS rats $(P<0.05, P<0.001$, two-sample $t$ test). At the same time, the latency of APs evoked by $100 \mathrm{pA}, 300 \mathrm{pA}$, and $500 \mathrm{pA}$ ramp current stimulation was significantly lower in the NMD + AMS group than in the CON + AMS group (Fig. 4G, $P<0.01$, Mann-Whitney test), indicating that the did not significantly alter the NE concentration compared with $\mathrm{CON}+$ ASS. C NMD + AMS markedly enhanced the NE concentration compared with CON + AMS. $* * P<0.01$, two-sample $t$ test.

neuronal excitability was enhanced in NMD rats after AMS treatment. 

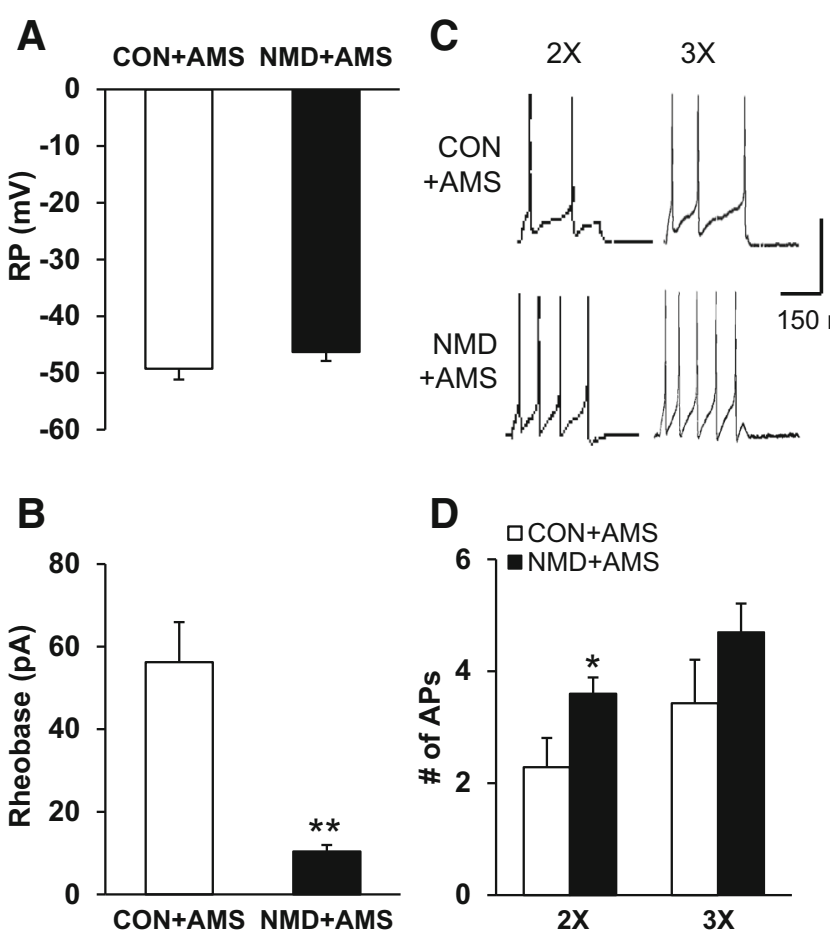

Fig. 4 NMD and AMS enhanced colon DRG neuronal excitability. A NMD + AMS depolarized the resting membrane potential (RP) in DRG neurons. B NMD + AMS resulted in a marked reduction of the rheobase in DRG neurons. C Representative traces of action potentials (APs) induced by $300 \mathrm{~ms}$ depolarizing current injection at $2 \times$ rheobase (left) and $3 \times$ rheobase (right) in DRG neurons from CON + AMS and NMD + AMS rats under current-clamp. D Bar graph showing a significant increase in average numbers of APs elicited by $2 \times$ rheobase current injection in NMD + AMS rats compared with CON + AMS rats. E Representative traces of APs induced by $1000 \mathrm{~ms}$ depolarizing current injection at $100 \mathrm{pA}, 300 \mathrm{pA}$,

\section{AMS Upregulates the Expression of $\boldsymbol{\beta}_{2}$ Adrenergic Receptors in DRGs}

Then we assessed the protein expression of adrenergic receptors beta 1, 2, $3\left(\beta_{1,2,3}\right)$ after AMS (Fig. 5A-C). The relative levels of $\beta_{2}$ receptor proteins were $1.17 \pm 0.10$ ( $n=$ 3 rats) and $1.11 \pm 0.14$ ( $n=4$ rats) from CON rats and NMD rats, respectively (Fig. 5E), with no significant difference between the two groups $(P>0.05$, two-sample $t$ test), which is consistent with the above behavioral studies. The relative values of $\beta_{2}$ receptor proteins were $0.81 \pm 0.13(n=4$ rats $)$ and $1.45 \pm 0.04(n=3$ rats $)$ in the $\mathrm{CON}+\mathrm{AMS}$ and NMD + AMS rats, respectively (Fig. 5B, $P<0.01$, two-sample $t$ test). However, there was no significant difference in $\beta_{1}$ and $\beta_{3}$ receptor protein levels between CON + AMS and NMD + AMS rats (Fig. 5A and C, $P>0.05$, two-sample $t$ test). Furthermore, the relative mRNA levels of $\beta_{2}$ receptor were $1.00 \pm 0.63$ $(n=5$ rats $)$ and $2.20 \pm 0.83$ ( $n=6$ rats $)$ in CON + AMS and NMD + AMS rats, respectively (Fig. 5D, $* P<0.05$,
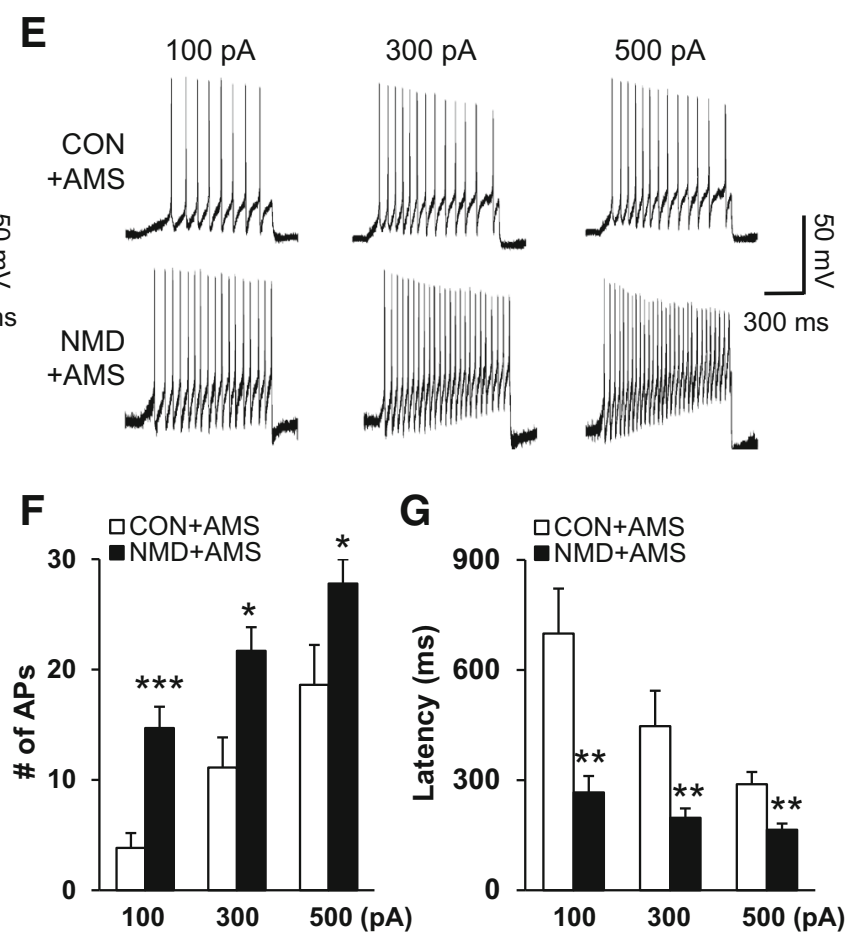

and $500 \mathrm{pA}$ ramp stimulation in DRG neurons from CON + AMS and NMD + AMS rats under current-clamp. F Bar graph showing a significant increase in average numbers of APs evoked by $100 \mathrm{pA}$, $300 \mathrm{pA}$, and $500 \mathrm{pA}$ ramp stimulation in NMD + AMS rats compared with CON + AMS rats. G Bar graph showing a significant decrease in latency of APs evoked by $100 \mathrm{pA}, 300 \mathrm{pA}$, and $500 \mathrm{pA}$ ramp stimulation in NMD + AMS rats compared with CON + AMS rats. NMD + AMS, $n=10$ cells, $* P<0.05, * * P<0.01, * * * P<$ 0.001 compared with CON + AMS, $n=8$ cells, Mann-Whitney test and two-sample $t$ test.

two-sample $t$ test). Besides, the relative values of $\beta_{2}$ receptor proteins were $0.71 \pm 0.07(n=4$ rats) and $0.92 \pm$ $0.02(n=4$ rats $)$ in CON + ASS and NMD + ASS rats, respectively (Fig. 5F) $(* P<0.05$, two-sample $t$ test).

\section{Treatment with $\beta_{2}$ Receptor Antagonist Reverses Visceral Hypersensitivity}

Next we determined whether AR antagonists can reverse chronic visceral hyperalgesia. Injection of PROP, a nonselective $\beta$ receptor antagonist, significantly reversed visceral hyperalgesia in a time-dependent manner (Fig. 6A, NS: $n=12$; PROP: $n=3, P<0.001$, compared with NS, Tukey post-hoc test following two-way repeated measures ANOVA). Further, injection of BUTO, a $\beta_{2}$ receptor antagonist, significantly raised the DT of NMD + AMS rats (Fig. $6 \mathrm{~B}, n=12$ rats/group, $P<0.05, P<0.001$, Tukey post-hoc test following two-way repeated measures ANOVA). However, injection of PHEN, an antagonist of $\alpha$ receptors, had no effect on the visceral hyperalgesia 

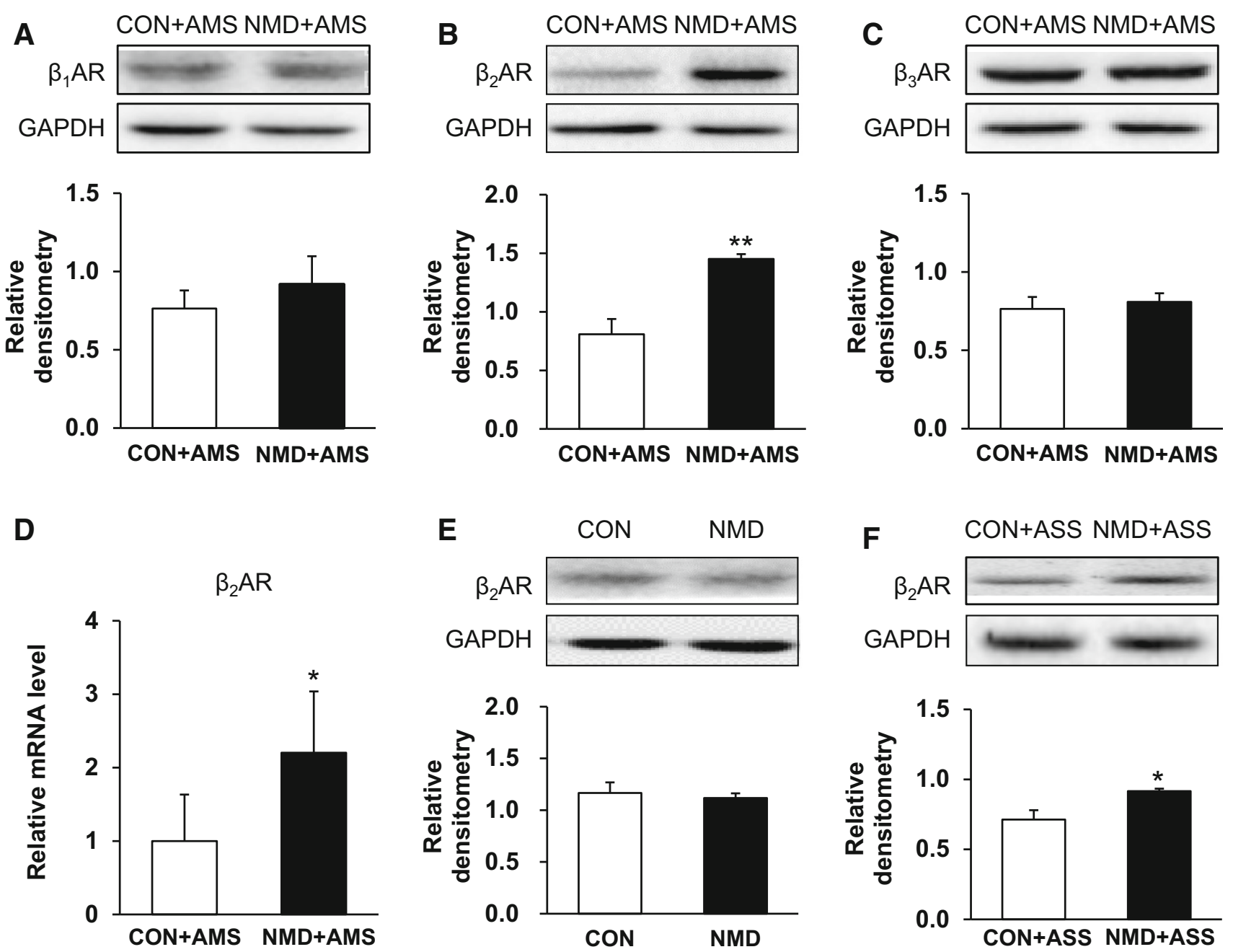

Fig. 5 Multiple stressors upregulated expression of $\beta_{2}$ adrenergic receptors (ARs) in DRGs of NMD rats at 6 weeks. A NMD + AMS did not change the expression of $\beta_{1}$ ARs in DRGs between $\mathrm{CON}+\mathrm{AMS}$ and NMD + AMS rats. B NMD + AMS significantly upregulated the expression of $\beta_{2}$ receptors when compared with CON + AMS groups. C NMD + AMS did not change the expression of $\beta_{3}$ receptors between $\mathrm{CON}+\mathrm{AMS}$ and $\mathrm{NMD}+\mathrm{AMS}$ rats.

induced by AMS (Fig. 6C, NS: $n=12$; PHEN: $n=3, P>$ 0.05, Tukey post-hoc test following two-way repeated measures ANOVA). In addition, there was no significant effect of BUTO at the same dose on the DT of CON + AMS rats (Fig. 6D, $n=5$ rat/group, $P>0.05$, Tukey posthoc test following two-way repeated measures ANOVA). These data suggested that $\beta_{2}$ receptors are involved in the development of the visceral hypersensitivity induced by AMS in NMD rats.

\section{Treatment with $\beta_{2}$ Receptor Antagonist Reverses Neuronal Hyperexcitability}

To investigate the role of $\beta_{2}$ receptors in NMD + AMS rats, we injected the $\beta_{2}$ receptor antagonist BUTO once
D NMD + AMS significantly enhanced the mRNA level of $\beta_{2}$ receptors when compared with CON + AMS groups. E NMD did not alter the expression of $\beta_{2}$ receptors when compared with CON groups at 6 weeks. F NMD + ASS significantly enhanced the expression of $\beta_{2}$ receptors when compared with CON + ASS groups. ${ }^{*} P<0.05$, $* * P<0.01$, two-sample $t$ test.

daily for 7 consecutive days to assess its influence on the excitability of colonic $\mathrm{T}_{13}-\mathrm{L}_{2}$ DRG neurons. The resting membrane potentials were $-40.78 \pm 0.38 \mathrm{mV}(n=9$ cells) in NS rats and $-49.23 \pm 1.11 \mathrm{mV}(n=13$ cells $)$ in BUTO rats (Fig. 7A, $P<0.001$, Mann-Whitney test). The average rheobases were $18 \pm 2 \mathrm{pA}$ ( $n=9$ cells) in NS rats and $33 \pm 4 \mathrm{pA}$ ( $n=13$ cells) in BUTO rats (Fig. 7B, $P<$ 0.01 , Mann-Whitney test). The numbers of APs in response to $2 \times$ and $3 \times$ rheobase current stimulation were significantly lower in DRG neurons after BUTO treatment (Fig. 7C, D, $P<0.05, P<0.001$, Mann-Whitney test and two-sample $t$ test). In addition, we counted the number and measured the latency of APs induced by 100 pA, 300 pA, and 500 pA ramp current stimulation (Fig. 7E-G). The numbers of APs were significantly decreased and their 

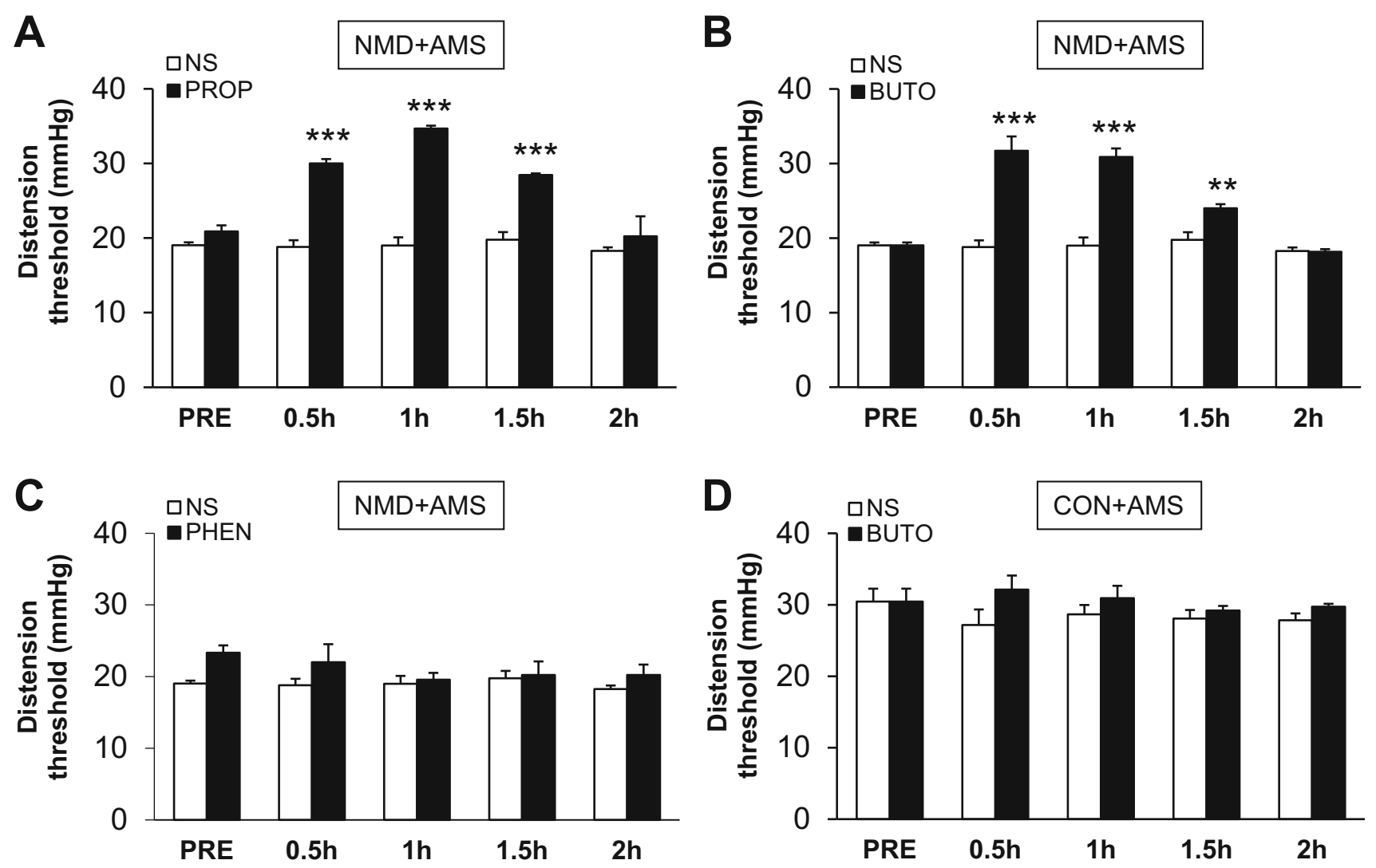

Fig. 6 Norepinephrine $\beta_{2}$ receptor antagonist reversed the visceral pain caused by NMD and AMS. A The NE $\beta$ receptor antagonist propranolol (PROP, $3 \mathrm{mg} / \mathrm{kg}$ ) significantly reduced the visceral hyperalgesia induced by NMD + AMS in a time-dependent manner. B The $\beta_{2}$ receptor antagonist butoxamine (BUTO, $5 \mathrm{mg} / \mathrm{kg}$ ) also reversed the visceral hyperalgesia induced by NMD + AMS in a

latency was markedly increased $(P<0.001$, MannWhitney test and two-sample $t$ test). These results demonstrated that BUTO decreased the neuronal excitability enhanced by NMD and AMS exposure.

\section{Discussion}

In the present experiments, we demonstrated that NMD rats exhibited reduced thresholds and increased abdominal withdrawal reflex scores to CRD when compared with age-matched control rats after exposure to multiple stressors as adults. This indicates that a combination of NMD and AMS exacerbated the symptoms by enhancing visceral hypersensitivity in NMD rats at the age of 6 weeks. This also supports an idea that NMD puts such rats at risk when they grew to the age of 6 weeks although these rats do not show any visceral hypersensitivity as reported previously [31]. Although these rats are more sensitive to multiple stressors at the age of 6 weeks than age-matched control rats, this does not mean that these NMD rats are sensitive

time-dependent manner. $\mathbf{C}$ The $\alpha$ receptor antagonist phentolamine (PHEN, $3 \mathrm{mg} / \mathrm{kg}$ ) had no effect on the visceral hyperalgesia induced by NMD + AMS. D BUTO $(5 \mathrm{mg} / \mathrm{kg})$ had no effect on CON + AMS rats. $* * P<0.01, * * * P<0.001$, Tukey post-hoc test following twoway repeated measures ANOVA. Note that the NS group in panels $\mathbf{A}-$ C used the same rats.

to any environmental stimulus, since the CRS alone did not induce any visceral response. Environmental stimuli have to reach a minimal threshold to induce visceral hypersensitivity. Although we did not define the minimal threshold for NMD rats, our data might have clinic relevance in that an adverse neonatal stimulus followed by adult stressors aggravates the symptoms of IBS or/and shortens the time window to induce visceral hypersensitivity.

The finding of enhanced visceral sensitivity was strongly supported by the enhanced neuronal excitability in NMD rats followed by AMS. By whole-cell patch clamp recording, we showed that the excitability of DRG neurons was remarkably enhanced in NMD rats followed by AMS when compared with that of age-matched control rats followed by AMS. Our electrophysiological data provide a cellular mechanism underlying the enhanced visceral pain behaviors. Of note is that the ionic basis for this enhanced cellular excitability needs further investigation.

In the present experiments, we focused on the mechanism by which AMS produced visceral hypersensitivity and neuronal hyperexcitability in NMD rats at the age of 6 

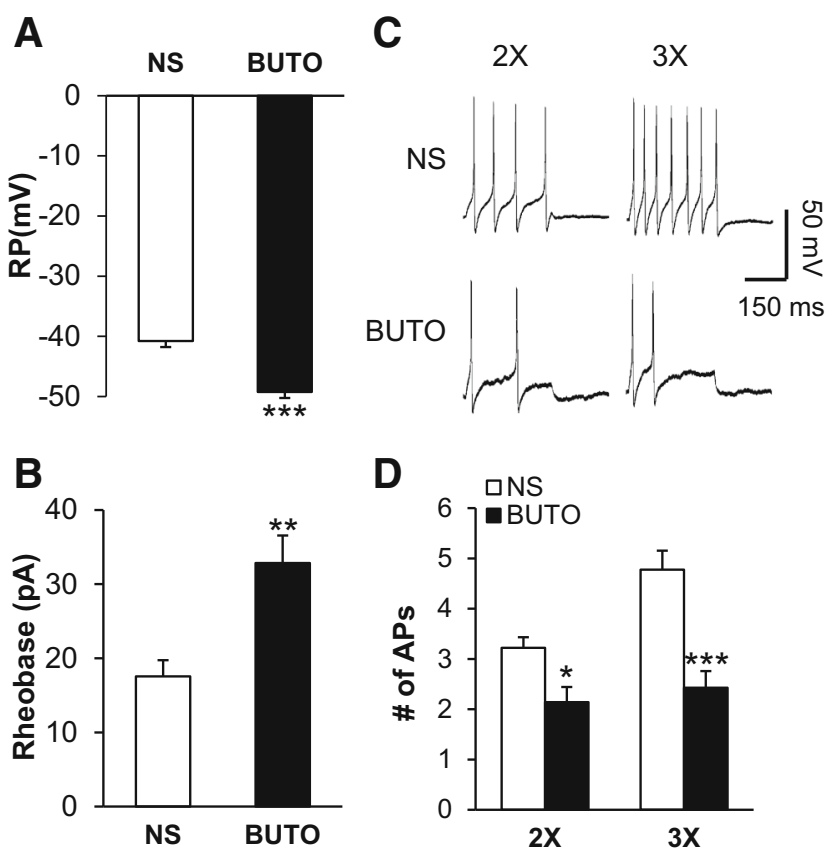

Fig. 7 A $\beta_{2}$ receptor antagonist reversed the enhanced excitability. A Administration of BUTO $(5 \mathrm{mg} / \mathrm{kg}$, once daily for 7 consecutive days) markedly reduced the resting potential (RP) increase induced by NMD + AMS. B BUTO significantly increased the rheobase decrease induced by NMD + AMS. C Representative traces of action potentials (APs) induced by $300 \mathrm{~ms}$ depolarizing current injection at $2 \times$ (left) and $3 \times$ (right) rheobase in DRG neurons from NS- and BUTO-treated NMD + AMS rats under current-clamp. D Bar graph showing a significant decrease in numbers of APs induced by $2 \times$ and $3 \times$ rheobase in BUTO-treated NMD + AMS rats, compared with NS-treated NMD + AMS rats. E Representative

weeks. A recent study has shown that chronic stress involves NE release, AR expression, and/or the activation of intermediates in AR-induced signaling, thus contributing to the pathology of many immune-mediated diseases [33]. Adrenergic activation is involved in neuropathic and inflammatory pain states $[34,35]$. Our previous studies showed that neonatal colonic inflammation or heterotypic intermittent stress increases the NE concentration in blood plasma without alterations in $\beta_{2}$ AR expression in DRGs $[14,20]$. A new finding in the present study was that AMS not only enhanced the plasma NE levels but also increased the expression of $\beta_{2}$ ARs in DRGs at 6 weeks. This discrepancy might be due to the different stimulus protocols used. The neonatal colonic inflammation model was established only by one colonic stimulus in the neonate. The heterotypic intermittent stress model was induced by stressors only in adulthood $[14,17]$. However, the present model was established by a combined stress protocol in both neonates and adults. This combination of stimuli might be more relevant to the clinic situation. Therefore, our study also provides a good animal model to better mimic the clinic situation in patients with IBS, thus
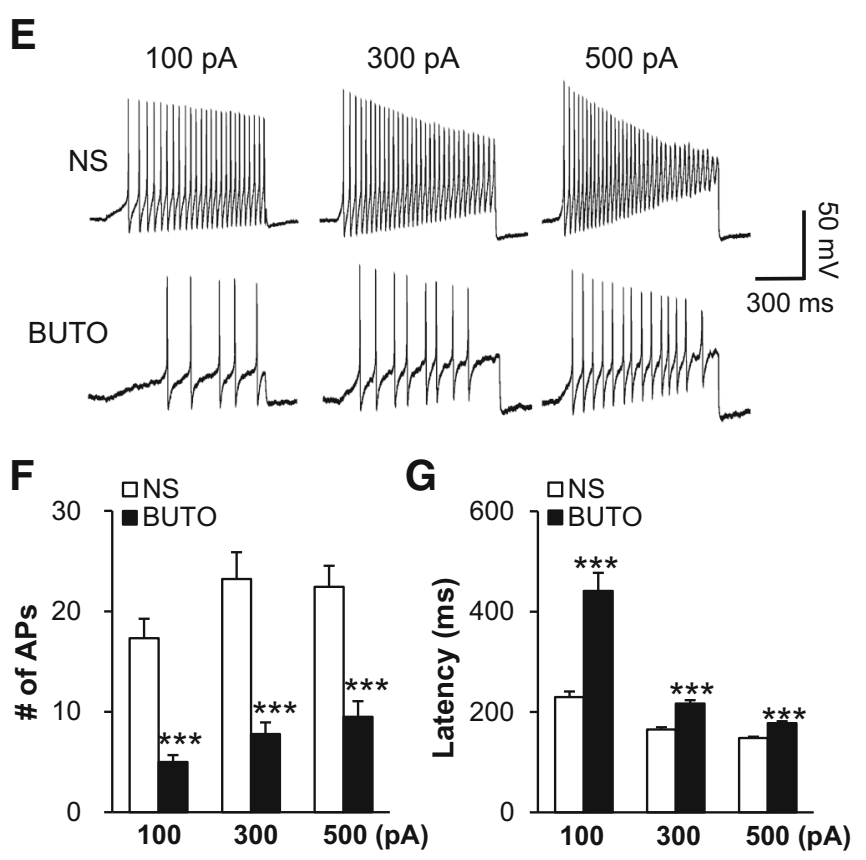

traces of APs induced by $1000 \mathrm{~ms}$ depolarizing current injection as $100 \mathrm{pA}, 300 \mathrm{pA}$, and $500 \mathrm{pA}$ ramps in DRG neurons from NS- and BUTO-treated NMD + AMS rats under current-clamp. F Bar graph showing a significant decrease in average numbers of APs evoked by $100 \mathrm{pA}, 300 \mathrm{pA}$, and $500 \mathrm{pA}$ ramps in BUTO-treated NMD + AMS rats, compared with NS-treated NMD + AMS rats. G Bar graph showing a significant increase in AP latency evoked by 100 pA, 300 $\mathrm{pA}$, and $500 \mathrm{pA}$ ramps in BUTO-treated NMD + AMS rats, compared with NS-treated NMD + AMS rats. BUTO, $n=13$ cells, $* P<0.05$, ** $P<0.01$, *** $P<0.001$ compared with NS, $n=9$ cells, Mann-Whitney test and two-sample $t$ test.

providing a better basis on which to investigate the mechanisms of visceral pain.

We showed that $\beta_{2}$ ARs played an important role in the peripheral nervous system. The expression of $\beta_{2}$ ARs at the protein and mRNA levels was remarkably upregulated in NMD rats with AMS while the expression of $\beta_{1}$ and $\beta_{3}$ ARs was not altered greatly. Inhibition of $\beta_{2}$ ARs by PROP or BUTO enhanced the CRD threshold in a time-dependent manner while inhibition of $\alpha$ adrenergic receptors by PHEN did not affect the CRD threshold. Furthermore, application of BUTO also reversed the enhanced hypersensitivity of DRG neurons. This anti-nociceptive effect was specific since BUTO did not have any effect on agematched control rats with AMS. These data demonstrated that enhanced $\mathrm{NE}$ and $\beta_{2}$ adrenergic signaling plays an important role in increasing the visceral hypersensitivity in rats with NMD and AMS. Of note is that the protein expression of $\beta_{2}$ ARs in NMD rats followed by ASS was also significantly increased. However, the NE level in the plasma was not altered remarkably. This may explain why the NMD rats followed by ASS did not show enhanced visceral sensitivity. In addition, we do not know whether 
AMS can exacerbate the visceral hypersensitivity of NMD rats at the age of 7 weeks, although NMD rats at this age already exhibit enhanced visceral hypersensitivity. In the present study, we only focused on the peripheral mechanisms; the central mechanisms, such as spinal synaptic plasticity [36], deserve further investigation.

In summary, we demonstrated that NMD followed by AMS increased visceral hypersensitivity in association with an elevation of the NE concentration in plasma, the expression of $\beta_{2}$ ARs in DRGs, and the neuronal excitability of colonic DRG neurons. Blockade of $\beta_{2}$ ARs attenuated visceral hypersensitivity to colorectal distension and neuronal hyperexcitability. Together with our previous studies, our data provide additional evidence to support the idea that the NE- $\beta_{2}$ signaling pathway plays an important role in the development of visceral hypersensitivity. This study might shed light on the pathogenesis of visceral hypersensitivity imposed by environmental stress during early and adult life. AR inhibitors might serve as alternates to relieve abdominal pain in patients with IBS.

Acknowledgements This work was supported by grants from the National Natural Science Foundation of China (81471137, 31730040, and 81500952) and the Priority Academic Program Development of Jiangsu Higher Education Institutions of China.

Conflict of interest The authors declare that they have no conflict of interest.

Open Access This article is distributed under the terms of the Creative Commons Attribution 4.0 International License (http:// creativecommons.org/licenses/by/4.0/), which permits unrestricted use, distribution, and reproduction in any medium, provided you give appropriate credit to the original author(s) and the source, provide a link to the Creative Commons license, and indicate if changes were made.

\section{References}

1. Canavan C, West J, Card T. Review article: the economic impact of the irritable bowel syndrome. Aliment Pharmacol Ther 2014, 40: 1023-1034.

2. Dupont HL. Review article: evidence for the role of gut microbiota in irritable bowel syndrome and its potential influence on therapeutic targets. Aliment Pharmacol Ther 2014, 39: 1033-1042.

3. Sayuk GS, Gyawali CP. Irritable bowel syndrome: modern concepts and management options. Am J Med 2015, 128: 817-827.

4. Ji RR. Recent progress in understanding the mechanisms of pain and itch: the second special issue. Neurosci Bull 2018, 34: 1-3.

5. Bai L, Wang X, Li Z, Kong C, Zhao Y, Qian JL, et al. Upregulation of chemokine cxcl12 in the dorsal root ganglia and spinal cord contributes to the development and maintenance of neuropathic pain following spared nerve injury in rats. Neurosci Bull 2016, 32: 27-40.

6. Kong X, Wei J, Wang D, Zhu X, Zhou Y, Wang S, et al. Upregulation of spinal voltage-dependent anion channel 1 contributes to bone cancer pain hypersensitivity in rats. Neurosci Bull 2017, 33: 711-721.

7. Gebhart GF, Bielefeldt K. Physiology of visceral pain. Compr Physiol 2016, 6: 1609-1633.

8. Drossman DA, Camilleri M, Mayer EA, Whitehead WE. AGA technical review on irritable bowel syndrome. Gastroenterology 2002, 123: 2108-2131.

9. Li L, Xie R, Hu S, Wang Y, Yu T, Xiao Y, et al. Upregulation of cystathionine beta-synthetase expression by nuclear factor-kappa B activation contributes to visceral hypersensitivity in adult rats with neonatal maternal deprivation. Mol Pain 2012, 8: 89.

10. Hu S, Xu W, Miao X, Gao Y, Zhu L, Zhou Y, et al. Sensitization of sodium channels by cystathionine beta-synthetase activation in colon sensory neurons in adult rats with neonatal maternal deprivation. Exp Neurol 2013, 248: 275-285.

11. Berrill JW, Sadlier M, Hood K, Green JT. Mindfulness-based therapy for inflammatory bowel disease patients with functional abdominal symptoms or high perceived stress levels. J Crohns Colitis 2014, 8: 945-955.

12. Labus J, Gupta A, Gill HK, Posserud I, Mayer M, Raeen H, et al. Randomised clinical trial: symptoms of the irritable bowel syndrome are improved by a psycho-education group intervention. Aliment Pharmacol Ther 2013, 37: 304-315.

13. Winston JH, Xu GY, Sarna SK. Adrenergic stimulation mediates visceral hypersensitivity to colorectal distension following heterotypic chronic stress. Gastroenterology 2010, 138: 294-304 e293.

14. Zhang C, Rui YY, Zhou YY, Ju Z, Zhang HH, Hu CY, et al. Adrenergic beta2-receptors mediates visceral hypersensitivity induced by heterotypic intermittent stress in rats. PLoS One 2014, 9: e94726.

15. Taguchi R, Shikata K, Furuya Y, Hirakawa T, Ino M, Shin K, et al. Selective corticotropin-releasing factor 1 receptor antagonist E2508 reduces restraint stress-induced defecation and visceral pain in rat models. Psychoneuroendocrinology 2017, 75: $110-115$.

16. Xu D, Gao J, Gillilland M, 3rd, Wu X, Song I, Kao JY, et al. Rifaximin alters intestinal bacteria and prevents stress-induced gut inflammation and visceral hyperalgesia in rats. Gastroenterology 2014, 146: 484-496.e4.

17. Wang Y, Qu R, Hu S, Xiao Y, Jiang X, Xu GY. Upregulation of cystathionine beta-synthetase expression contributes to visceral hyperalgesia induced by heterotypic intermittent stress in rats. PLoS One 2012, 7: e53165.

18. Hieble JP, Bondinell WE, Ruffolo RR Jr. Alpha- and betaadrenoceptors: from the gene to the clinic. 1. Molecular biology and adrenoceptor subclassification. J Med Chem 1995, 38: 3415-3444.

19. Ruffolo RR Jr, Bondinell W, Hieble JP. Alpha- and betaadrenoceptors: from the gene to the clinic. 2. Structure-activity relationships and therapeutic applications. J Med Chem 1995, 38: 3681-3716.

20. Zhu L, Zhao L, Qu R, Zhu HY, Wang Y, Jiang X, et al. Adrenergic stimulation sensitizes TRPV1 through upregulation of cystathionine beta-synthetase in a rat model of visceral hypersensitivity. Sci Rep 2015, 5: 16109.

21. Ruffolo RR Jr, Hieble JP. Alpha-adrenoceptors. Pharmacol Ther 1994, 61: 1-64.

22. Aley KO, Martin A, McMahon T, Mok J, Levine JD, Messing RO. Nociceptor sensitization by extracellular signal-regulated kinases. J Neurosci 2001, 21: 6933-6939.

23. Wang YJ, Li XF, Ding F, Shu Q, Song LJ, Yu X, et al. Noradrenaline regulates substance $\mathrm{P}$ release from rat dorsal root ganglion neurons in vitro. Neurosci Bull 2011, 27: 300-306.

24. Zhang J, Halm ST, Halm DR. Adrenergic activation of electrogenic $\mathrm{K}+$ secretion in guinea pig distal colonic epithelium: 
involvement of beta1- and beta2-adrenergic receptors. Am J Physiol Gastrointest Liver Physiol 2009, 297: G269-G277.

25. Khasar SG, Lin YH, Martin A, Dadgar J, McMahon T, Wang D, et al. A novel nociceptor signaling pathway revealed in protein kinase C epsilon mutant mice. Neuron 1999, 24: 253-260.

26. Khasar SG, McCarter G, Levine JD. Epinephrine produces a betaadrenergic receptor-mediated mechanical hyperalgesia and in vitro sensitization of rat nociceptors. J Neurophysiol 1999, 81: 1104-1112.

27. Skeberdis VA. Structure and function of beta3-adrenergic receptors. Medicina (Kaunas) 2004, 40: 407-413.

28. Juif PE, Salio C, Zell V, Melchior M, Lacaud A, PetitDemouliere $\mathrm{N}$, et al. Peripheral and central alterations affecting spinal nociceptive processing and pain at adulthood in rats exposed to neonatal maternal deprivation. Eur J Neurosci 2016, 44: 1952-1962.

29. Wang Q, Zhu H, Zou K, Yuan B, Zhou YL, Jiang X, et al. Sensitization of $\mathrm{P} 2 \mathrm{X} 3$ receptors by cystathionine beta-synthetase mediates persistent pain hypersensitivity in a rat model of lumbar disc herniation. Mol Pain 2015, 11: 15.

30. Yuan B, Tang WH, Lu LJ, Zhou Y, Zhu HY, Zhou YL, et al. TLR4 upregulates CBS expression through NF-kappaB activation in a rat model of irritable bowel syndrome with chronic visceral hypersensitivity. World J Gastroenterol 2015, 21: 8615-8628.

31. Hu S, Xiao Y, Zhu L, Li L, Hu CY, Jiang X, et al. Neonatal maternal deprivation sensitizes voltage-gated sodium channel currents in colon-specific dorsal root ganglion neurons in rats. Am J Physiol Gastrointest Liver Physiol 2013, 304: G311-G321.

32. Pertovaara A. The noradrenergic pain regulation system: a potential target for pain therapy. Eur J Pharmacol 2013, 716: 2-7.

33. Padro CJ, Sanders VM. Neuroendocrine regulation of inflammation. Semin Immunol 2014, 26: 357-368.

34. Choi JW, Kang SY, Choi JG, Kang DW, Kim SJ, Lee SD, et al. Analgesic effect of electroacupuncture on paclitaxel-induced neuropathic pain via spinal opioidergic and adrenergic mechanisms in mice. Am J Chin Med 2015, 43: 57-70.

35. Wang S, Zhu HY, Jin Y, Zhou Y, Hu S, Liu T, et al. Adrenergic signaling mediates mechanical hyperalgesia through activation of P2X3 receptors in primary sensory neurons of rats with chronic pancreatitis. Am J Physiol Gastrointest Liver Physiol 2015, 308: G710-G719.

36. Zhou LJ, Liu XG. Glial activation, a common mechanism underlying spinal synaptic plasticity? Neurosci Bull 2017, 33: 121-123. 